\title{
The Characteristic Analysis of Weak Current Sensor
}

\author{
Su Chengzhi (Corresponding author) \\ College of Mechanical and Electric Engineering \\ Changchun University of Science and Technology, Changchun 130022, China \\ Tel: 86-137-5688-9054Ｅ-mail: chengzhi_su@126.com \\ Qiao Kuipu \\ College of Mechanical and Electric Engineering \\ Changchun University of Science and Technology, Changchun 130022, China
}

Tel: 86-159-4836-6510 E-mail: qiao2056@126.com

Xu Yudong

College of Mechanical and Electric Engineering

Changchun University of Science and Technology, Changchun 130022, China

E-mail: xuyudongchongqing@163.com

Wang Enguo
College of Mechanical and Electric Engineering
Changchun University of Science and Technology, Changchun 130022, China
E-mail: 64951930@qq.com

Li Zhenhui

College of Mechanical and Electric Engineering

Changchun University of Science and Technology, Changchun 130022, China

E-mail:1zr95@sohu.com

Received:January 4, 2012

doi:10.5539/mas.v6n4p63
Accepted: March 7, $2012 \quad$ Published: April 1, 2012

URL: http://dx.doi.org/10.5539/mas.v6n4p63

\begin{abstract}
The electrical equipment loss caused by the lightning and power's increasing demand is reduced by monitoring the extent of the surge protector failure through measuring its leakage current on-line. Proposed a method of monitoring the surge protector's failure degree online based on mutual inductance current sensor, established its mathematical model, analyzed the influence of coil turns, load resistance and stray capacitance on the amplitude-frequency and phase-frequency characteristic. And used impact and sinusoidal signal to composite a motivic signal to test the optimized current sensor's time-domain response feature by simulation. Experiments show: while the range of measured current is $0.2 \sim 9 \mathrm{~mA}$, the mutual inductance current sensor designed can resist the impact of $50 \mathrm{kA}$ high current, the current measurement error can be less than $0.11 \mathrm{~mA}$, meet requirement of the leakage current on-line monitoring.
\end{abstract}

Keywords: Mutual inductance coil, Current sensor, Impact resistance, Online monitoring

\section{Introduction}

Lightning is a serious natural disaster, not only caused casualties, but also resulted in the damage of the buildings, electronic equipments, transmission lines and communication equipments. The surge caused by the electricity 
increasing demand can damage the transmission line of electronic equipment (Wang Q. P., Tian R. M. \& He Q., 2006). In order to reduce and avoid these losses and damage, installed surge protector for transient overvoltage protection, its principle is that leading the surge into the ground to limit the transient over-voltage in the range equipment or system can withstand, which flees into power and signal transmission lines, avoid the protected equipment or system is damaged by the impact. Whether the surge protector is working properly or not relate to the electrical equipment safety directly, so it is very important to monitor the operation timely and effectively in the lightning protection system and power system.

When the surge protector is working normally, its leakage current is very small, usually only tens of microamps, but if not, the leakage current will be increased over $1 \mathrm{~mA}$. To achieve the surge protector on-line monitoring, it is required resisting the impact of high current $50 \mathrm{kA}$, and the current measurement error is less than $0.2 \mathrm{~mA}$. Now, there are usually two ways to monitor the failure (deterioration or damage) of the current surge protector: manual inspection and leakage current detection (Cui M. J. \& Han C. X., 2002). The first method needs intensive labor, it is not convenience to detect the equipment that is installed in the field or high place, so it can't detect the failure of surge protector timely. The second method usually detect the leakage current of surge protector with hall current sensor or giant magneto-resistance sensor, the measurement method has a simple principle, wide bandwidth, and can resist strong impact. It demands the measurement accuracy of current sensor no less than 0.2 $\mathrm{mA}$ at least for measuring $1 \mathrm{~mA}$ leakage current accurately, and the equivalent magnetic field strength is $2 \times 10^{-9} \mathrm{~T}$, the Hall current sensor measurement range is $10^{-7} \sim 10 \mathrm{~T}$ (Zhang W. N. \& Du Z., 1996), the giant magneto-resistance sensor measurement range is $10^{-8} \sim 10^{8}$ T (Qian Z., Zhang X. \& Tao X. Y., 2007), thus the second method is difficult to monitor the working status of surge protector accurately.

This paper proposed a method for measuring the leakage current of surge protector and monitoring its failure degree with mutual inductance current sensor. A mutual inductance mathematical model will be established to analyze the principle of resisting large current impact, and make an anti-impact optimization on the current sensor structure parameters based on the principle.

\section{Erect Sensor Model}

The measurement principle of Surge protector leakage current is shown in Figure 1 (Su C. Z., Xu, Y. D. \& Wang N. G., 2011). The structure of mutual inductance current sensor is shown in Figure 1(a) (Yang C. Z., Chen G. Q., 2011), which is composed by the core and the coil that is around the core, the core material uses high permeability permalloy, $i_{1}(t)$ is the measured current of current sensor, the back-end uses low-noise op amp. The structure equivalent circuit is shown in Figure 1(b), in which, $i_{1}(t)$ is the measured current with one turn, $M$ is the mutual inductance of the coil, $L$ is the coil inductance, $R_{L}$ is the coil equivalent resistance, $C_{0}$ is the coil equivalent stray capacitance, $R$ is the coil load resistance, $e(t)$ is the potential generated by mutual inductance, $u(t)$ is the voltage drop across the coil load resistance.

From the Figure 1(b), the relationship between the measured current signal $i_{1}(t)$ and the potential $e(t)$ generated by coil mutual inductance can be given by

$$
e(t)=M \frac{d i_{1}(t)}{d t}
$$

in the equation (1), $M$ is calculated from

$$
M=\mu \frac{N S}{l}
$$

$M$ - mutual inductance

$N$-coil turns

$S$-magnetic core cross-section

$\mu$-magnetic permeability

$l$-length of magnetic path

From the Kirchhoff voltage law, the equation of the current sensor equivalent circuit is given by

$$
e(t)=L C_{0} \frac{d^{2} u(t)}{d t}+\left(\frac{L}{R}+R_{L} C_{0}\right) \frac{d u(t)}{d t}+\left(1+\frac{R_{L}}{R}\right) u(t)
$$

assuming initial input is 0 , we can get the transfer function as following after doing laplace transform on equation (2), 


$$
G(s)=\frac{U(s)}{I_{1}(s)}=\frac{R}{N} \frac{s}{R C_{0} s^{2}+\left(1+\frac{R_{L} C_{0} R}{L}\right) s+\frac{R_{L}+R}{L}}
$$

its amplitude-frequency characteristic is given by

$$
G(f)=|G(j 2 \pi f)|=\frac{1}{C_{0} N} \frac{2 \pi f}{\sqrt{\left(\frac{R+R_{L}}{R C_{0} L}-(2 \pi f)^{2}\right)^{2}+\left(\frac{1}{R C_{0}}+\frac{R_{L}}{L}\right)^{2} \cdot(2 \pi f)^{2}}}
$$

its phase-frequency characteristic is given by

$$
\varphi(f)=\arctan \frac{R+R_{L}-R C_{0} L \cdot(2 \pi f)^{2}}{\left(L+R_{L} C_{0} R\right) \cdot(2 \pi f)}
$$

\section{Simulaition}

Analyzing the amplitude-frequency and phase frequency response characteristics with simulation, without loss of generality, we assume that the current sensor parameters are as follows:

$\mu=\mu_{r} \mu_{0}, \quad \mu_{r}=10^{5}, \quad \mu_{0}=4 \pi \times 10^{-7} \frac{H}{m}, \mathrm{D}=42 \mathrm{~mm}, \mathrm{~d}=24 \mathrm{~mm}, C_{0}=0.24 n F, R_{L}=375 \Omega, R=1 \mathrm{~K} \Omega, h=9 \mathrm{~mm}$,

$S=8.1 \times 10^{-5} \mathrm{~m}^{2}, \quad \mathrm{l}=\frac{\mathrm{D}+\mathrm{d}}{2} \cdot \pi=0.10362 \mathrm{~m}, \quad L=\mu \frac{N^{2} \cdot S}{l}=9.8 \times 10^{-5} \mathrm{~N}^{2} \mathrm{H}$

When $N$ gets10, 100 and 1000 turns respectively, from equations (4) and (5), we can obtained the curves of amplitude-frequency characteristic and phase-frequency characteristic that are shown in Figure 2.

From the Figure 2, it is found that the turns' number $N$ is greater, the passband is wider, the passband gain also called sensitivity is lower, the phase variation is more intense, and the lower limit of passband cutoff frequency is lower, but $N$ has little effect on the upper limit of passband cutoff frequency. While $N$ is smaller, the passband is narrower, the passband gain is higher, and the phase change linearity is better.

Defined $N=1000$ turns, $C_{0}=0.24 n F$, from the equation (4) and (5), we can get the curves of amplitude-frequency and phase-frequency characteristic that are shown in Figure 3 when $R$ selects $10 \Omega, 1 \mathrm{~K} \Omega, 1 \mathrm{M} \Omega$.

From the Figure 3, the load resistance $R$ is greater, the sensitivity of the sensor is higher, and the pass band is narrower, but the center frequency is unchanged. Therefore, to improve the sensitivity of the current sensor, the load resistance cannot be too small; to have a certain bandwidth, load resistance cannot be too large.

Defined $N=1000$ turns, $\mathrm{R}=1 \mathrm{~K} \Omega$, from the equation (4) and (5), we can get the curves of amplitude-frequency and phase-frequency characteristic that are shown in Figure 4 when $C_{0}$ selects $0.24 n F, 10 n F, 1000 n F$.

From the Figure 4, we can know that the stray capacitance $C_{0}$ affect the upper limit frequency $f_{H}$ of the passband cutoff frequency of the sensor amplitude-frequency characteristic, while $C_{0}$ increases, $f_{H}$ decreases, but has little effect on the sensor sensitivity and lower limit frequency $f_{L}$, because the equivalent resistance of the coil is too small to affect the sensitivity and lower frequency in the variation range of the stray capacitance and the spectrum of the partial discharge signals is so wide. In practice, there is not only stray capacitance existing in the two ends of the load resistance, but also the ground capacitance existing in signal transmission cable, which has a greater effect on the sensor than the stray capacitance, thus, the cable must be considered when analyzing or designing the sensor.

In order to ensure the current sensor has high measurement sensitivity and anti-impact ability while measuring the $50 \mathrm{~Hz}$ leakage current, we select $N=1000$ turns, $\mathrm{R}=1 \mathrm{~K} \Omega, C_{0}=100 \mathrm{nF}$ after analyzing by simulation, other parameters are the same as given above, in the following, the parameters do not change if no special instruction.

Assuming current sensor input current is $i_{1}(t)=I_{m} \delta(t)+I_{n} \sin \omega t$, where $I_{m} \delta(t)$ the impact is current, $I_{m}$ is the impact current amplitude, taken as $50 \mathrm{kA}, I_{n} \sin \omega t$ is the leakage current, $I_{n}$ is the leakage current amplitude, taken as $0.2 \mathrm{~mA}$, do Laplace transform on input current, from the equation $Y(s)=G(s) I_{1}(s)$, we can obtain the result:

$$
Y(s)=\frac{1}{N C_{0}} \cdot \frac{s}{s^{2}+\left(\frac{1}{R C_{0}}+\frac{R_{L}}{L}\right) s+\frac{R+R_{L}}{R C_{0} L}} \cdot\left(I_{m}+I_{n} \frac{\omega}{s^{2}+\omega^{2}}\right)
$$


From the equation $\xi=\frac{L+R C_{0} R_{L}}{2 \sqrt{R L C_{0}\left(R+R_{L}\right)}}$, we can calculate the damp $\xi>1$, it is a underdamping system, transferring the formula (6) with inverse laplace transform, the result is

$$
\begin{aligned}
y(t)= & L^{-1}[Y(s)] \\
= & \frac{I_{m} R L \omega_{n}}{2 N\left(R+R_{L}\right) \sqrt{\xi^{2}-1}}\left[\omega_{n}\left(\xi+\sqrt{\xi^{2}-1}\right) e^{-\left(\xi+\sqrt{\xi^{2}-1}\right) \omega_{n} t}\right. \\
& \left.\left.-\omega_{n}\left(\xi-\sqrt{\xi^{2}-1}\right) e^{-\left(\xi-\sqrt{\xi^{2}-1}\right) \omega_{n} t}\right)\right] \\
& -\frac{I_{n} R L \omega}{4 N\left(R+R_{L}\right) \xi \sqrt{\xi^{2}-1}}\left(e^{-\left(\xi-\sqrt{\xi^{2}-1}\right) \omega_{n} t}-e^{-\left(\xi+\sqrt{\xi^{2}-1}\right) \omega_{n} t}\right) \\
& +\frac{I_{n} R L \omega_{n}}{2 \xi N\left(R+R_{L}\right)} \sin \omega t
\end{aligned}
$$

where $\omega_{n}=\sqrt{\frac{R+R_{L}}{R C_{0} L}}, \xi=\frac{L+R C_{0} R_{L}}{2 \sqrt{R L C_{0}\left(R+R_{L}\right)}}$, the formula (7) is shown in Figure 5, it is found that the shock wave attenuates to zero at $0.005 \mathrm{~s}$, then the output is a sine wave, the sensor can attenuate the impact current quickly and measure the $0.2 \mathrm{~mA}$ leakage current accurately.

\section{Experiment}

The $50 \mathrm{~Hz}$ sine wave got by signal generator is used to simulate leakage current, and the $50 \mathrm{kA}$ impact current is generated by the impact current generator. First, load analog leakage current into the current sensor separately when doing the experiment, while the analog leakage current amplitude is $4.79 \mathrm{~mA}$, the response waveform shown in Figure 6; then load the analog leakage current and the impact current into the current sensor at the same time, and record the data before and after the experimental that is shown in Table 1, in which $i$ represents analog leakage current, $u_{1}$ represents the average of peak voltage before impact, $i_{1}$ represents the leakage current in the current sensor before impact, $e_{1}$ represents the measurement error before impact, $u_{2}$ represents the average of peak voltage after impact, $i_{2}$ represents the leakage current in the current sensor after impact, $e_{2}$ represents the measurement error after impact. According to the data in Table 1, respectively, we can draw the relationship curves between the analog leakage current and the average of the peak voltage before and after the impact, which are shown in Figure 7 and Figure 8. Where the dashed line formed by the symbol '*' represents the curve drawn with the actual measurement data, solid line represents a straight line fitted by the least-squares, the curve formed by the ' $x$ ' represents the actual measurement error curve. We can figure out the current sensor sensitivity and the current measurement equation by the fitting curve before and after the impact.

From the least-squares fitting straight line, the current sensor sensitivity before the impact is given by

$$
k_{1}=\frac{\Delta i_{1}}{\Delta u_{1}}=0.7073 \mathrm{~mA} / \mathrm{V}
$$

the current measurement equation before the impact is given by

$$
i_{1}=k_{1} u_{1}+b_{1}=0.7073 u_{1}-0.2649
$$

from the least-squares fitting straight line, the current sensor sensitivity after the impact is given by

$$
k_{2}=\frac{\Delta i_{2}}{\Delta u_{2}}=0.7070 \mathrm{~mA} / \mathrm{V}
$$

the current measurement equation after the impact is given by

$$
i_{2}=k_{2} u_{2}+b_{2}=0.7070 u_{2}-0.2634
$$

From the equation (9) and (11), figure out the current sensor measurement current before and after the impact, compared with analog leakage current and obtain the measurement error $e_{1}$ and $e_{2}$ that is shown in Table 1 . Before the impact the current maximum measurement error is $0.102 \mathrm{~mA}$, after the impact the current maximum measurement error is $0.108 \mathrm{~mA}$, after the impact the measurement error is $0.006 \mathrm{~mA}$ bigger than before the impact, but it still meets the design requirement that leakage current measurement error is less than $0.2 \mathrm{~mA}$. 
Experiments show that: in the current measurement range $0.2 \sim 9 \mathrm{~mA}$, the current sensor current measurement error is less than $0.11 \mathrm{~mA}$, and it can resist the impact of $50 \mathrm{kA}$ high current.

\section{Conclusions}

Experiments show that: the mutual inductance current sensor designed can resist the impact of $50 \mathrm{kA}$ high current in the current measurement range $0.2 \sim 9 \mathrm{~mA}$, and current measurement error is less than $0.11 \mathrm{~mA}$.

The structure of the sensor designed is simple, it does not change the original wiring of the measured device, belongs to a non-contact measurement, not consumes the energy of the measured source, and the load effect is small.

The research provides an effective method for the leakage current online monitoring of the surge protector, and has certain significance for the measurement of the anti-impact alternating weak signal.

\section{References}

Cui, M. J., \& Han, C. X. (2002). Lightning protection and application of surge protection device. Yunnan Electric Power, 38, 73-76.

Qian, Z., Zhang, X., \& Tao, X. Y. (2007). The Characteristics' Test and Analysis of Giant Magneto-resistance Current Sensor. High voltage apparatus, 43, 340-345.

Su, C. Z., Xu, Y. D., \& Wang, N. G. (2011). Detection Method of Leakage Current of Surge Protective Device. Low Voltage Apparatus, 10, 49-52.

Wang, Q. P., Tian, R. M., \& He, Q. (2006). Primary analysis on the thunder damage and thunder observation. Thunder prevention and Observation Center of Shanxi Province, 75, 31-33.

Yang, C. Z., \& Chen, G. Q. (2011). Design of sensor based onmutual inductance principle. Journal of Mechanical \& Electrical Engineering, 28, 590-593.

Zhang, W. N., \& Du, Z. (1996). The application of hall current sensor in insulation on-line monitoring and measurement. High voltage engineering, 22, 30-32.

Table 1. Experiment data measured by the current sensor

\begin{tabular}{|l|l|l|l|l|l|l|}
\hline$l / \mathbf{m A}$ & $u_{1} / \mathbf{V}$ & $i_{1} / \mathbf{m A}$ & $e_{1} / \mathbf{m A}$ & $u_{2} / \mathbf{V}$ & $i_{2} / \mathbf{m A}$ & $e_{2} / \mathbf{m A}$ \\
\hline 0.152 & 0.61 & 0.169 & 0.017 & 0.60 & 0.161 & 0.009 \\
\hline 0.471 & 1.16 & 0.558 & 0.087 & 1.15 & 0.550 & 0.079 \\
\hline 0.952 & 1.66 & 0.912 & 0.040 & 1.65 & 0.903 & 0.049 \\
\hline 1.410 & 2.22 & 1.308 & 0.102 & 2.26 & 1.334 & 0.076 \\
\hline 1.916 & 3.02 & 1.874 & 0.042 & 3.01 & 1.865 & 0.051 \\
\hline 2.383 & 3.62 & 2.298 & 0.085 & 3.62 & 2.296 & 0.087 \\
\hline 2.865 & 4.36 & 2.821 & 0.044 & 4.38 & 2.833 & 0.032 \\
\hline 3.354 & 5.23 & 3.437 & 0.083 & 5.24 & 3.441 & 0.087 \\
\hline 3.836 & 5.88 & 3.896 & 0.06 & 5.91 & 3.915 & 0.079 \\
\hline 4.312 & 6.49 & 4.328 & 0.016 & 6.47 & 4.311 & 0.001 \\
\hline 4.792 & 7.12 & 4.773 & 0.019 & 7.24 & 4.855 & 0.063 \\
\hline 5.271 & 7.96 & 5.367 & 0.096 & 7.98 & 5.379 & 0.108 \\
\hline 5.756 & 8.59 & 5.813 & 0.057 & 8.59 & 5.810 & 0.054 \\
\hline 6.252 & 9.20 & 6.244 & 0.008 & 9.18 & 6.227 & 0.025 \\
\hline 6.731 & 9.82 & 6.683 & 0.048 & 9.80 & 6.665 & 0.066 \\
\hline 7.218 & 10.55 & 7.199 & 0.019 & 10.57 & 7.210 & 0.008 \\
\hline 7.695 & 11.16 & 7.631 & 0.064 & 11.16 & 7.627 & 0.068 \\
\hline 8.173 & 11.89 & 8.147 & 0.026 & 11.87 & 8.129 & 0.044 \\
\hline 8.654 & 12.67 & 8.699 & 0.045 & 12.67 & 8.694 & 0.004 \\
\hline 9.156 & 13.32 & 9.158 & 0.002 & 13.32 & 9.154 & 0.002 \\
\hline
\end{tabular}




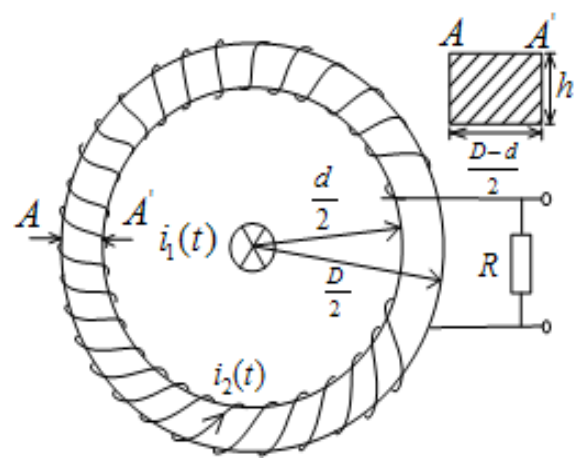

(a) The structure of current sensor

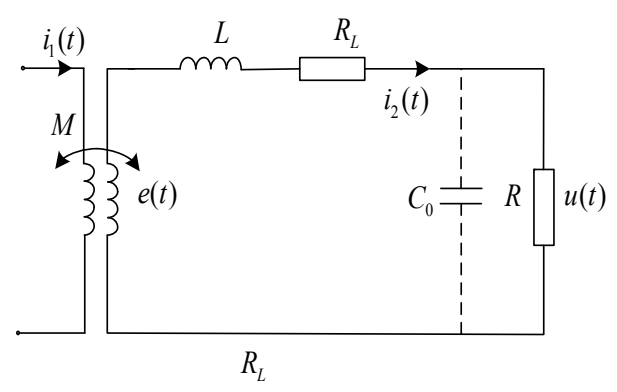

(b) The current sensor equivalent circuit

Figure 1. Principle of the current sensor

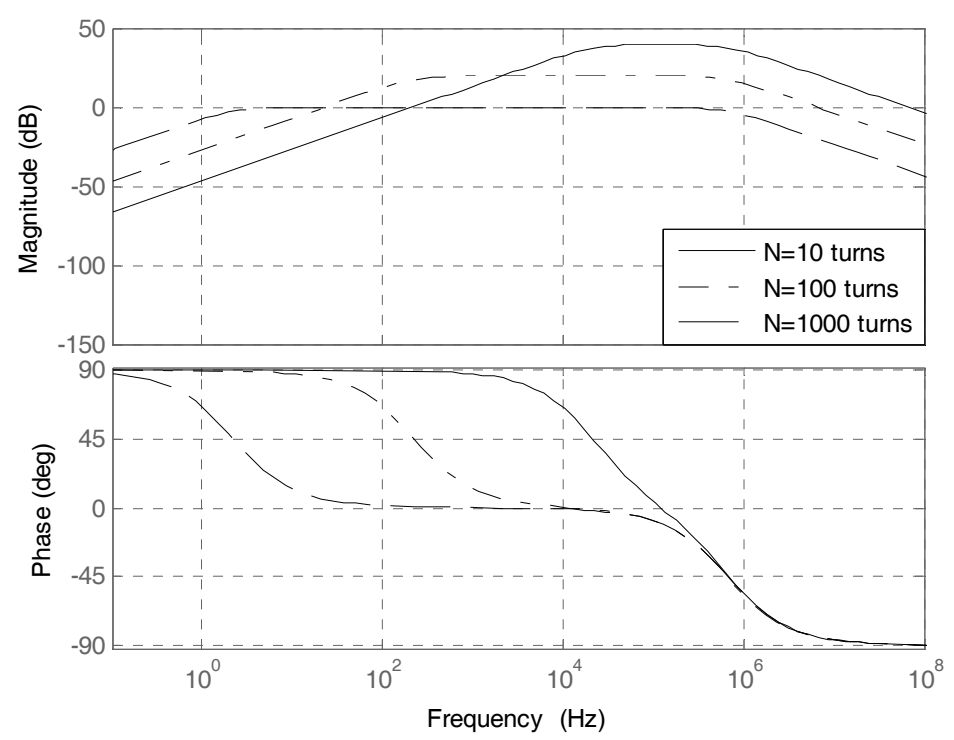

Figure 2. The influence of coil turns on the amplitude-frequency and phase-frequency characteristic

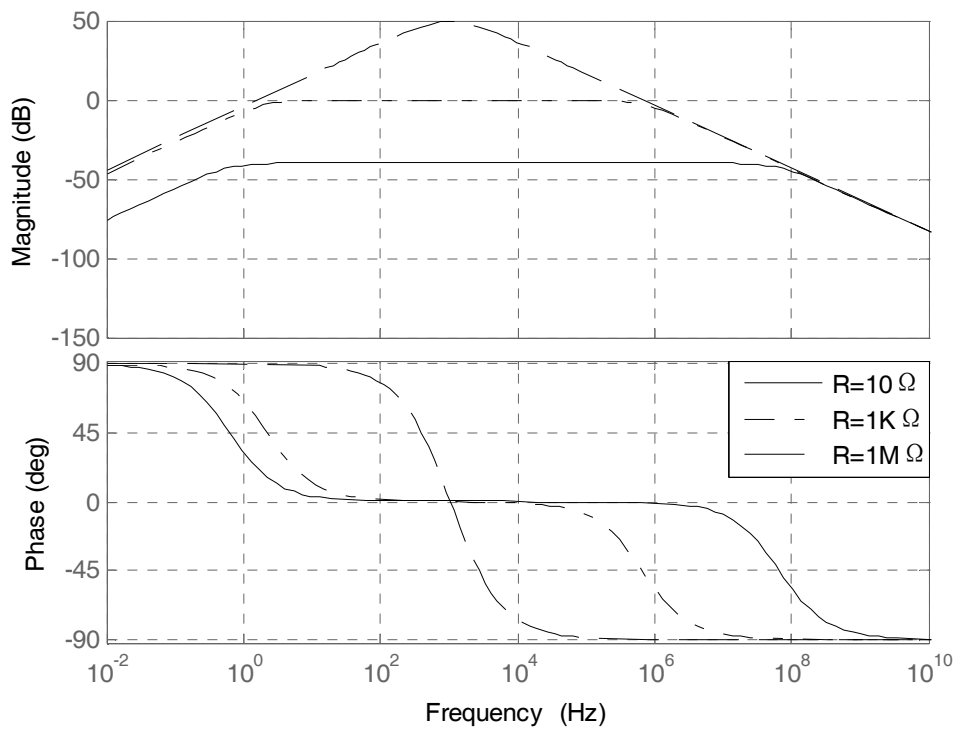

Figure 3. The influence of load resistance on the amplitude-frequency and phase-frequency characteristic 


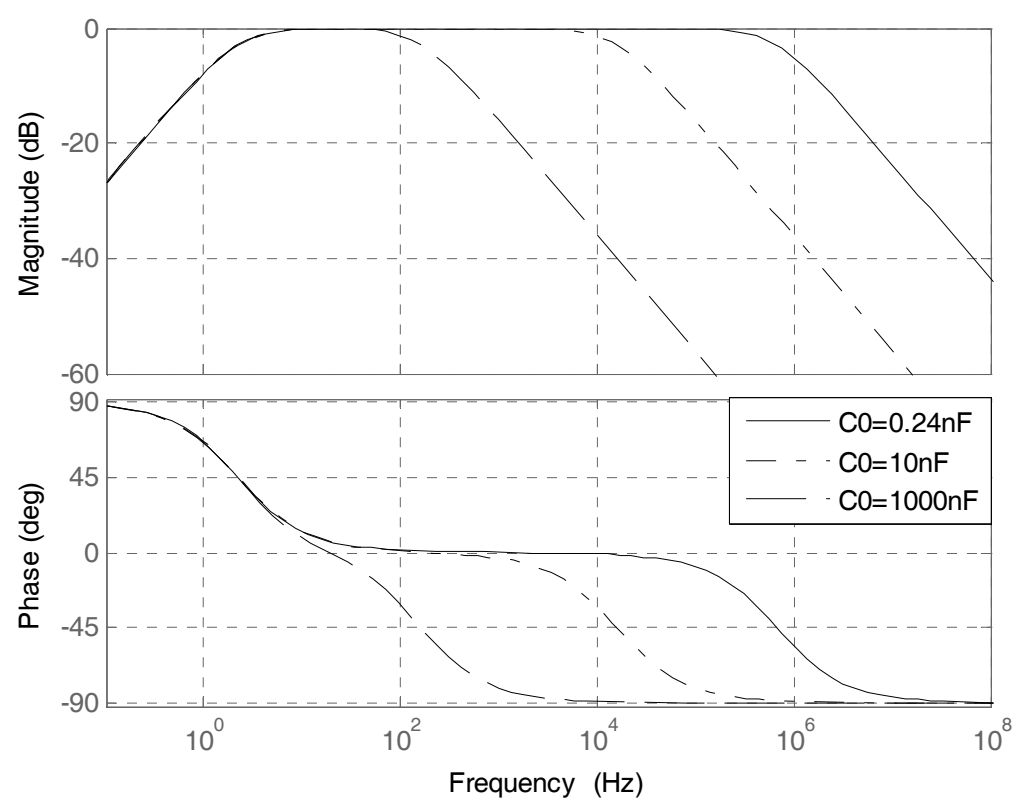

Figure 4. The influence of stray capacitance turns on the amplitude-frequency and phase-frequency characteristic

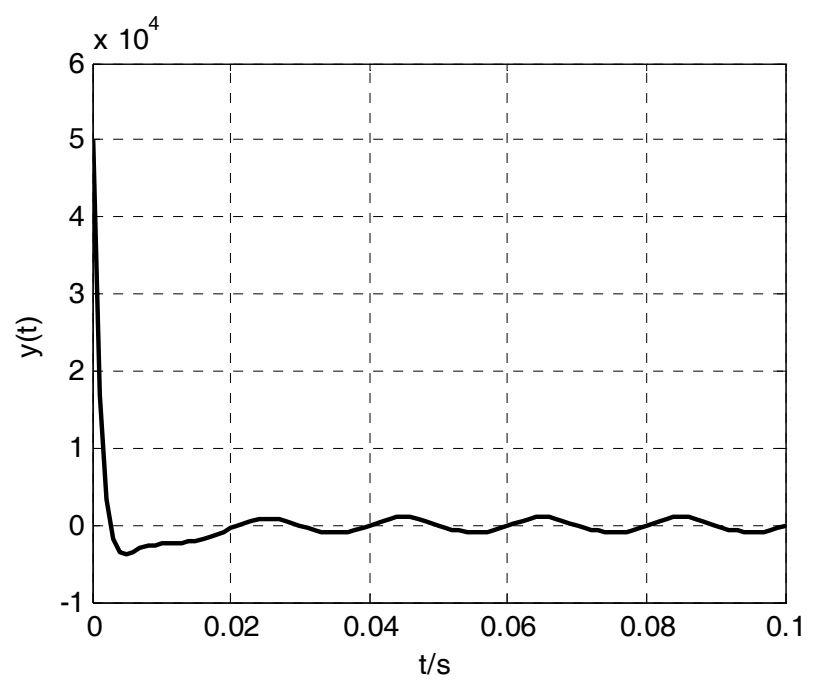

Figure 5. Response curve of the current sensor on the composite signal

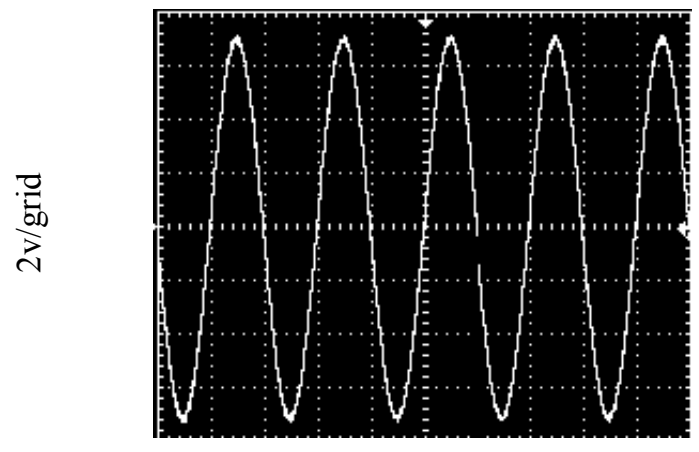

$10 \mathrm{~ms} /$ gri

Figure 6. Response waveform of the current sensor 


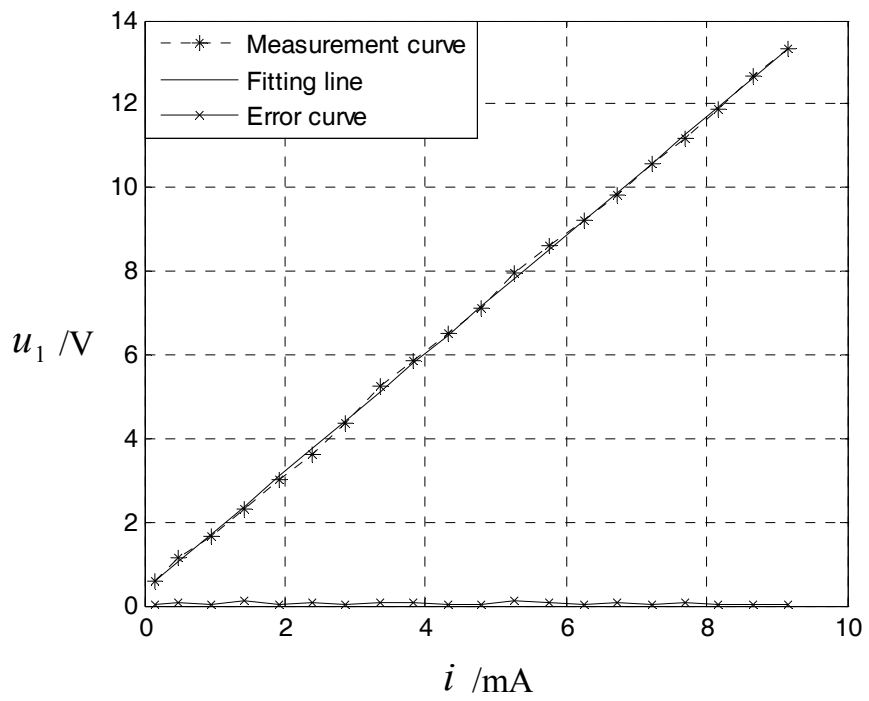

Figure 7. The curve between analog leakage current and the average of peak voltage before the impact

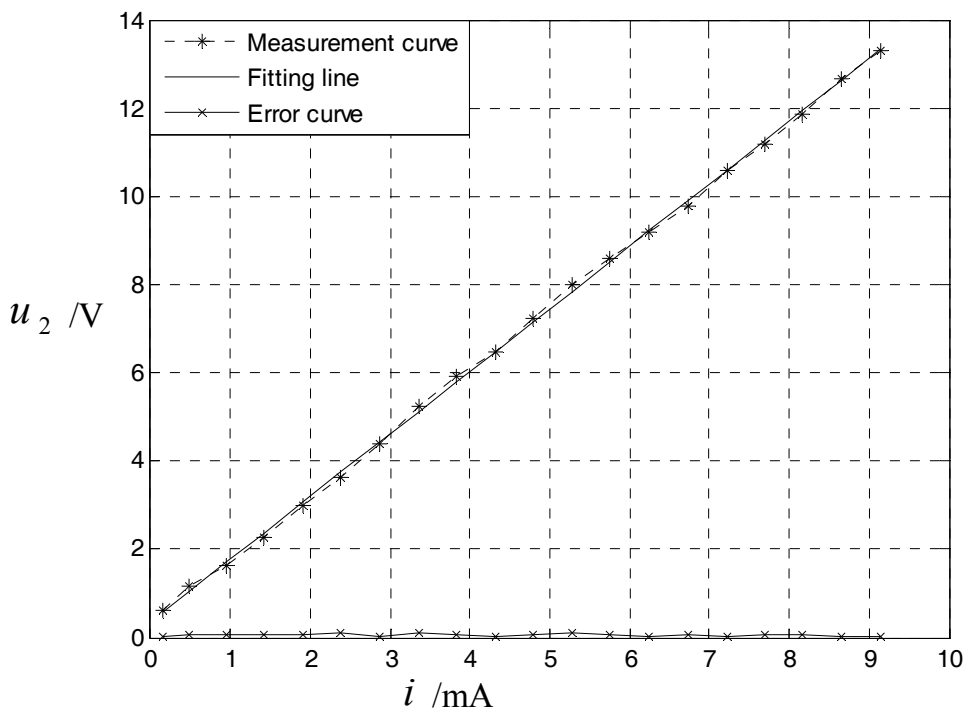

Figure 8 . The curve between analog leakage current and the average of peak voltage after the impact 